\title{
73 Intracranial extension of suppurative otitis media
}

A Can occur by bone erosion by cholesteatoma.

B May be through the petrosquamous suture line.

C Via the labyrinth may extend along the aqueduct of the cochlea.

D Through the mastoid emissary vein is possible.

E Along a dehiscent facial canal is rare.

\section{Complications of suppurative otitis media}

A Include retropharyngeal abscess.

B Rarely involve the middle cranial fossa because of the resistance of the tegmen antri and tympani.

C May give a positive Tobey-Ayer test.

D Are more likely to give rise to otogenic intracranial hypertension with the left ear.

E Include Luc's abscess.

\section{Secretory otitis media (SOM)}

A is most prevalent in the second decade.

B In the infant is commonly due to an abnormal eustachian tube.

C Is more likely in the presence of large infected adenoids.

D Infection is an aetiological factor.

E May be consequent upon inadequate antibiotic therapy for acute otitis media. 